\title{
The Sri Lankan prototype of a negative pressure wound therapy device
}

\section{K T D Kahaduwa ${ }^{1}$, T D Gooneratne ${ }^{1}$, S M Wijeyaratne ${ }^{1}$}

(Index words: wound, negative pressure wound therapy, device)

Ceylon Medical Journal 2011; 56: 31-32

The growing burden of non healing wounds and the associated rise in healthcare cost is a global issue. Such problems pose even greater challenges to developing countries such as Sri Lanka. Newer treatment modalities that may overcome delays in healing cannot be overlooked.

Negative pressure wound therapy (NPWT) is a noninvasive system that creates a localised, controlled subatmospheric (negative) pressure environment conducive for wound healing [1]. Although the concept was born in the early 1950s, it was Morykwas and Argenta who demonstrated that application of $100-150 \mathrm{mmHg}$ negative pressure through a closed system to an open wound increased the microvascular blood flow by 4 times the baseline value [2]. Further research has pointed to the stimulatory effect of microstrain on cellular mitogenesis, angiogenesis, and amplification of growth factors [3]. It has also been proposed that healing is accelerated by active evacuation of excess interstitial fluid, lowering of the heightened capillary after-load and a qualitative dilution of contained microcontaminants, bacteria, and proinflammatory cytokines $[3,4]$. Several studies have successfully demonstrated acceleration of healing with negative pressure and this has led to its large scale clinical application [5].
In a practical sense, clinically safe and effective negative pressures cannot be maintained on wound surfaces without the use of commercially available devices such as V.A.C. ${ }^{\circledR}$ Therapy, (KCI, San Antonio,Tex), Versatile $1^{\mathrm{TM}}$ (BlueSky Medical, Carlsbad, Calif) and RENASYSTM EZ (Smith \& Nephew). Such devices are yet to be marketed in Sri Lanka owing to prohibitive costs. We report the use of intermittent NPWT on resistant wounds in Sri Lanka using a locally assembled, easily affordable device.

The device consisted of an electric vacuum pump, valves, a reservoir, pressure gauge, tubing and a drainage bottle connected serially (Figure 1 ). The individual components were purchased locally and the device assembled at a cost of SLR 30,000. The drainage tube from the device is placed within the gauze dressing, which is applied onto the wound surface, and the wound is sealed air tight with a layer of 'Opsite' adhesive sheet. The device has to be manually switched off when the desired negative wound pressure is obtained. The initial negative pressure is subsequently lost gradually, due to the slow leak of air into the system. Hence, the device needs to be switched on again to regain the desired negative pressure.

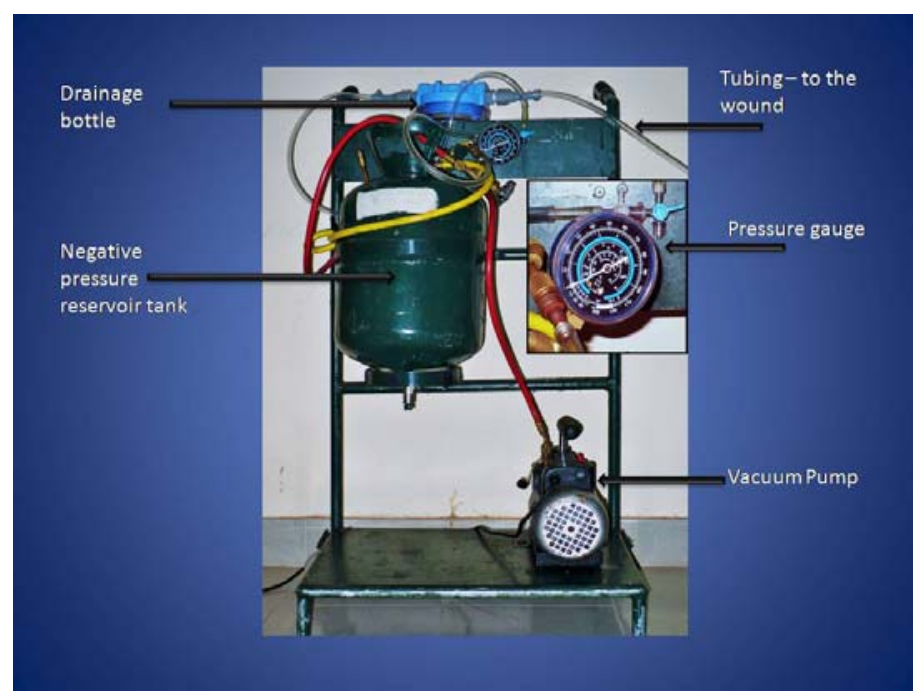

Figure 1. The negative pressure wound therapy device.

${ }^{1}$ Department of Surgery, University of Colombo, Sri Lanka.

Correspondence: KTDK, e-mail <thusitha_kahaduwa@yahoo.co.uk>. Received 13 April and accepted 30 October 2010. Competing interests: none declared. 
We report results from application of NPWT to 10 consenting hospitalised patients with resistant lower extremity wounds that had been present for more than 4 weeks (median 12.5 weeks) and had not shown signs of healing on standard wound care, i.e. debridement and local application of saline, dilute povidone iodine gauze dressings, hydrocolloid gel and polyurethane foam. Their median age was 46 years (range $38-58$ ) and 80\% were men. All were diabetic with heavy bacterial colonisation but without systemic sepsis. Wound dressings were changed every 2-4 days based on the amount and purulence of the exudates. One died from myocardial infarction before the wound healed. The remaining 9 demonstrated a dramatic increase in healthy granulation with reduction in purulence. The wounds were ready for split skin grafting after a mean duration of 13.5 days of therapy.

Automation of the process, linking pressure sensing with the switching on process will add further value to this device. Such locally developed devices would provide an opportunity for wider use of NPWT in Sri Lanka.

\section{References}

1. Armstrong DG, Attinger CE, Boulton AJ, et al. Guidelines regarding negative wound therapy (NPWT) in the diabetic foot. Ostomy Wound Management 2004; 50: 3S-27S.

2. Argenta LC, Morykwas MJ. Vacuum-assisted closure: a new method for wound control and treatment: clinical experience. Annals of Plastic Surgery 1997; 38: 563-77.

3. McNulty A, Schmidt M, Feeley T, Kieswetter K. Effects of negative pressure wound therapy on fibroblast viability, chemotactic signaling, and proliferation in a provisional wound (fibrin) matrix. Wound Repair and Regeneration 2007; 15: 838-46.

4. Gray M, Peirce B. Is negative pressure wound therapy effective for the management of chronic wounds? Journal of Wound Ostomy and Continence Nursing 2004; 31: 101-5.

5. Noble-Bell G, Forbes A. A systematic review of the effectiveness of negative pressure wound therapy in the management of diabetes foot ulcers. International Wound Journal 2008; 5: 233-42. 